\title{
A Comparative Analysis of Evolutionary Algorithms for Synthesis of Scanned Linear Array of Mutually Coupled Parallel Dipole Antennas
}

\author{
Hemant Patidar, Gautam Kumar Mahanti, and Ramalingam Muralidharan
}

\begin{abstract}
This paper presents a comparative analysis of three evolutionary algorithms, namely, Backtracking Search Algorithm, Cuckoo Search Algorithm and Artificial Bee Colony Algorithms for synthesis of a scanned linear array of uniformly spaced parallel half wavelength dipole antennas. Here, antenna parameters, namely Side Lobe Level, reflection coefficient and wide null depth are taken into consideration for comparison between algorithms. In addition to it, statistical parameters, namely best fitness value, mean and standard deviation of the fitness values obtained from algorithms are compared. Mutual coupling that exists among the antenna elements is included in obtaining radiation patterns and the self-impedances along with the mutual impedances are calculated by induced Electro-Motive Force method. Two different examples are shown in this paper to validate the effectiveness of the utilized approach. Although, this approach is applied to a linear array of dipole antennas; this can be utilized for other array geometries as well.
\end{abstract}

Keywords-Antenna array, comparative analysis, Evolutionary algorithms, inverse fast Fourier transform, scanning

\section{INTRODUCTION}

$\mathrm{T}$ HE advantages of Antenna arrays [1], namely steering of the main beam as well as producing high directivity makes them quite visible in both the commercial and military applications. Although various geometries of antenna arrays are available, a very commonly used one is the linear antenna array that produces a sufficiently narrow beam along with wide null depth in required angles. Several antenna array synthesis techniques dealing with various design parameters including null placements have been discussed in the literature [2-8].

Other than the above mentioned advantages that the antenna array produces, null steering [2-5] property finds itself in many applications to minimize the degradation of signal-to-noise ratio per interference in radar as well as other communication systems. Adaptive nulling using only phase with a genetic algorithm [2], various methods for null control [3] and their implications on antenna pattern are discussed with prior importance to null placements. Null synthesis by phase control for antenna arrays [4] and pattern nulling using perturbation of phases [5] also shows the importance of null placement in radiation patterns. Since mutual coupling plays an important role in degradation of radiation patterns, Antenna array synthesis technique have concentrated on the inclusion of this

H. Patidar and G.K. Mahanti are with the department of Electronics and Communication Engineering, National Institute of Technology, Durgapur, India (e-mail: hemantpatidar08@gmail.com, gautammahanti@yahoo.com).

$\mathrm{R}$ Muralidharan is with Department of Electrical and Computer Engineering, Caledonian College of Engineering, Sultanate of Oman (e-mail: muralidharan@caledonian.edu.om). coupling in various dimensions, namely, design of phasedifferentiated reconfigurable array antennas with a minimum value of the dynamic range ratio [6], Particle Swarm Optimization (PSO) versus Genetic Algorithms (GA) for phased array synthesis [7] and Mutual Coupling in Phased Arrays [8].

In this paper, the authors have considered the inclusion of nulls in certain directions during the generation of the radiation patterns. In a phased array, all the individual elements are fed coherently with a constant scan angle and all elements' phase shifters help in scanning the beam to desired directions. Because of mutual coupling effects, the input impedance of the element of an array is different from its impedance value when the same element is detached. Moreover, this difference between impedance values varies with the scan angle of the array. This impedance differences creates unwanted scenarios in the radiation patterns. For direction finding in small arrays, this results in great extent of phase errors that diminishes the angle of arrival estimations and for a larger array, it affects the side lobe level (SLL) also. The task of steering the array radiation pattern with the inclusion of mutual coupling is indeed a difficult task. Literature review [9-13] has reported about synthesis of antenna arrays in presence of mutual coupling.

A quick look at the synthesis of the arrays shows the support that evolutionary algorithms produced in them. These evolutionary algorithms have played a vital role in generation of the desired radiation patterns by generating the current distributions. Few of the algorithms are quoted below. Backtracking Search Algorithm (BSA), a new population based evolutionary algorithm, has been successfully used by $\mathrm{P}$. Civicioglu [14-15] for numerical optimization problems. The Cuckoo Search algorithm (CS), based on the strategy of searching of cuckoo birds to lay their eggs in an appropriate host bird nest has been discussed in [16-19]. Artificial Bee Colony (ABC) algorithm, which is based on a distinctive intelligent dealing of honeybee swarms has been described in [20-22]. The reason for choosing these algorithms in this paper is that they have succeeded in providing solutions to many optimization problems in the past. Further, in order to speed up the process of synthesis, the Inverse Fast Fourier Transform (IFFT) relationship that exists between the array factor and element excitations has been successfully used for synthesis of array patterns [23-25]. Combination of GA and Fast Fourier transform (FFT) for Array Synthesis is described in [23]. Fast 
low SLL synthesis for larger sized planar array antennas employing successive FFTs of Array Factor [24], low side lobe pattern synthesis employing iterative Fourier techniques are detailed in [25].

In this paper, the aim is to obtain the optimum set of current amplitude distribution using the algorithms (BSA, CS and $\mathrm{ABC}$ ) that will satisfy the goal. In addition to it, the performance of these algorithms based on the antenna radiation pattern parameters as well as the statistical parameters are compared. Self-impedances and mutual impedances are calculated by induced Electro-Motive Force (EMF) method. Mutual coupling matrix is first calculated and then stored in a mat file. To calculate the voltage distribution across each antenna, the program calls this mat file at every iteration and multiplies it with current excitation distribution along with a constant scan angle. This method along with calculation of array factor by means of IFFT reduces the computational time to a great extent. In addition to the above, effort is taken to make sure that there exists a better level of matching between the antenna and the corresponding feed network is available by minimizing maximum reflection coefficient to a value nearby to the specified.

\section{APPROACH}

A linear antenna array constructed of parallel halfwavelength thin dipole antennas with uniform spacing $d$ between the elements is considered along the $y$-axis. Elements are placed side-by-side as shown in Fig. 1. Using pattern multiplication, the free space far-field pattern of an array constructed of similar elements is the product of the element pattern and the pattern of an array of isotropic point sources. This linear organized array of dipoles is located in free space. The implications of ground plane are not taken into consideration.

The free space far-field pattern $F P(u)$ in vertical plane (y$z$ plane) [1] is given by Eq. (1):

$$
F P(u)=A F(u) \times E P(u)=\left[\sum_{n=1}^{N} I_{n} e^{j u_{n}} e^{j(n-1) k d u}\right] \times E P(u)
$$

where $n$ refers to the element number, $j$ is the imaginary quantity, $d$ is the inter element spacing which is taken as $0.5 \lambda$, $k=2 \pi / \lambda$ is the wave number, $\lambda$ is the wavelength, $u=\sin \theta, \theta$ being the polar angle of far-field measured from $z$-axis $\left(-90^{\circ}\right.$ to $\left.+90^{\circ}\right), u_{n}=-(n-1) k d u_{0}=$ progressive phase, $u_{0}=\sin \theta_{0}$ is the scan angle, $I_{n}$ is the amplitude (current) of the $n^{- \text {th }}$ element, $N$ is the total number of elements, $A F(u)$ is the array factor and $E P(u)$ is the element pattern of each $x$-directed horizontal half-wave length dipole antenna.
The element pattern of the dipole antenna is given by the equation below:

$$
E P(u)=\frac{\cos \left(\frac{\pi}{2} u\right)}{\sqrt{1-u^{2}}}
$$

and when the dipole antenna axis is along $x$-axis then $u$ is given by:

$$
u=\sin \theta \cos \phi
$$

Here, the element pattern of horizontal half wavelength dipole antenna is assumed omnidirectional in $y-z$ plane $\left(\phi=90^{\circ}\right.$ plane), i.e. $E P(u)=1$.

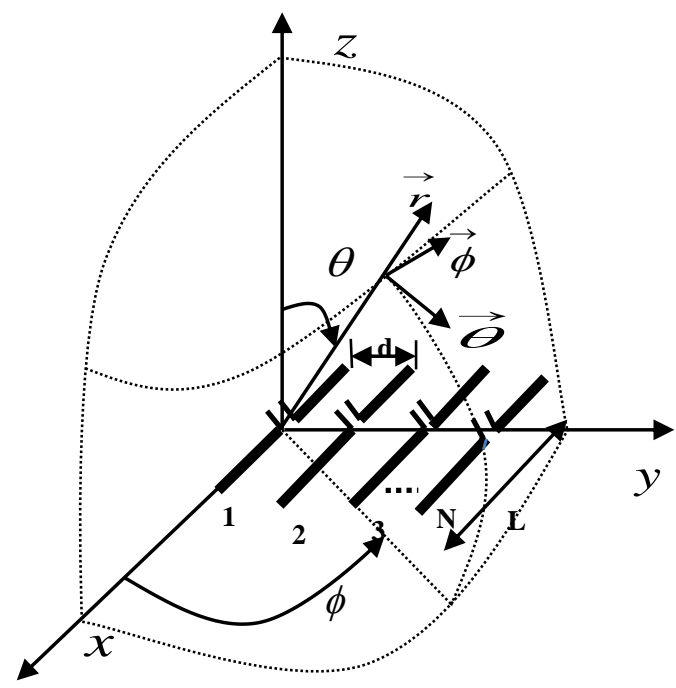

Fig.1. Figure of linear array of $\mathrm{x}$-directed parallel half-wave length center fed dipole antennas along y-axis.

The voltage across the $n^{\text {th }}$ dipole $[1,13]$ is given by:

$$
V_{n}=Z_{n n} I_{n}+\sum_{m \neq n} Z_{n m} I_{m}
$$

where $\mathrm{Z}_{n n}$ is the self-impedance of dipole $n$ and $\mathrm{Z}_{n m}$ is the mutual impedance between dipoles $n$ and $m$. The active scan impedance $[1,13]$ of dipole $n, \mathrm{Z}_{n}{ }^{\mathrm{A}}$ is given by:

$$
Z_{n}^{A}=\left|V_{n} / I_{n}\right|=Z_{n n}+\sum_{m \neq n} Z_{n m}\left(I_{m} / I_{n}\right)
$$

In the end, the maximum active scan impedance $\left(Z_{n, \max }^{A}\right)$ is calculated among all elements.

The voltage reflection coefficient is a parameter that describes the effect of impedance discontinuity or mismatch in the network. It is the ratio of the reflected voltage to that of the forward voltage. Assuming that the characteristic impedance $\left(Z_{o}\right)$ of the network that feeds is equal to $50 \mathrm{ohms}$, the maximum reflection coefficient $\left(R C_{\max }\right)$ at the input of $n^{\text {-th }}$ dipole antenna [1] is given by 
$R C_{\text {max }}=\left[\frac{\left|Z_{n}^{A}\right|_{\max }-Z_{o}}{\left|Z_{n}^{A}\right|_{\max }+Z_{o}}\right]$

When $\left|Z_{n}^{A}\right|_{\max }=Z_{o}$, it refers to the condition of the full matching of the load. In other words, no loss is there in the network and complete power is obtained at the load. This is an ideal situation that rarely happens. Voltage Standing Wave Ratio (VSWR) of 2.0 means approximately $10 \%$ of the power is reflected to source. A value of reflection coefficient $=0.33$ will give the VSWR value of 2.0. In the real world, VSWR of more than 2.0 is not considered.

The problem now is to obtain the set of current amplitude (excitations) of all the array elements using BSA, CS and ABC algorithms that minimizes the following fitness function and provide the desired results:

$$
\text { Fitness }=\left[k_{1} \times F_{1}^{2}+k_{2} \times F_{2}^{2}+k_{3} \times F_{3}\right]
$$

Where

$$
\begin{gathered}
F_{1}=\left\{\begin{array}{l}
S L L_{o}-S L L_{d}, \text { if } \rightarrow S L L_{o}>S L L_{d} \\
0, \text { if } \rightarrow S L L_{o} \leq S L L_{d}
\end{array}\right. \\
F_{2}=\left\{\begin{array}{l}
W N_{\text {max }}^{o}-W N_{\text {max }}^{d}, \text { if } \rightarrow W N_{\text {max }}^{o}>W N_{\text {max }}^{d} \\
0, \text { if } \rightarrow W N_{\text {max }}^{o} \leq W N_{\text {max }}^{d}
\end{array}\right. \\
F_{3}=\left\{\begin{array}{l}
R C_{\text {max }}^{o}-R C_{\text {max }}^{d}, \text { if } \rightarrow R C_{\text {max }}^{o}>R C_{\text {max }}^{d} \\
0, \text { if } \rightarrow R C_{\text {max }}^{o} \leq R C_{\text {max }}^{d}
\end{array}\right.
\end{gathered}
$$

The coefficients $k_{1}, k_{2}$ and $k_{3}$ are the relative weights given to each term in Eq. (8). The terms $S L L_{o}$ and $S L L_{d}$ are the obtained and the desired values of SLL, $W N_{\max }^{o}$ and $W N_{\max }^{d}$ are the obtained and the desired values of the maximum wide null depth and $R C_{\text {max }}^{o}$ and $R C_{\text {max }}^{d}$ are the obtained and the desired values of maximum reflection coefficient respectively. IFFT has been used for the sole purpose of reducing the time or speeding up the complete process. Since this has been already proved in [23-25], comparison has not been done on the process with IFFT. However, processing time has been compared for all the used algorithms.

\section{OVERVIEW OF BSA, CS AND ABC}

BSA proposed by P. Civicioglu [14-15], is an evolutionary algorithm which for generating a trial population uses a strategy that contains two new operators' crossover and mutation. The strategy for controlling the amplitude of the search-direction matrix and search-space boundaries increases the exploration and exploitation capabilities. BSA has two control parameters, namely dim_rate, which in this problem is kept to 1 and the other is a scale factor, set as $3^{*}$ randn, with randn being random normal distribution [15].

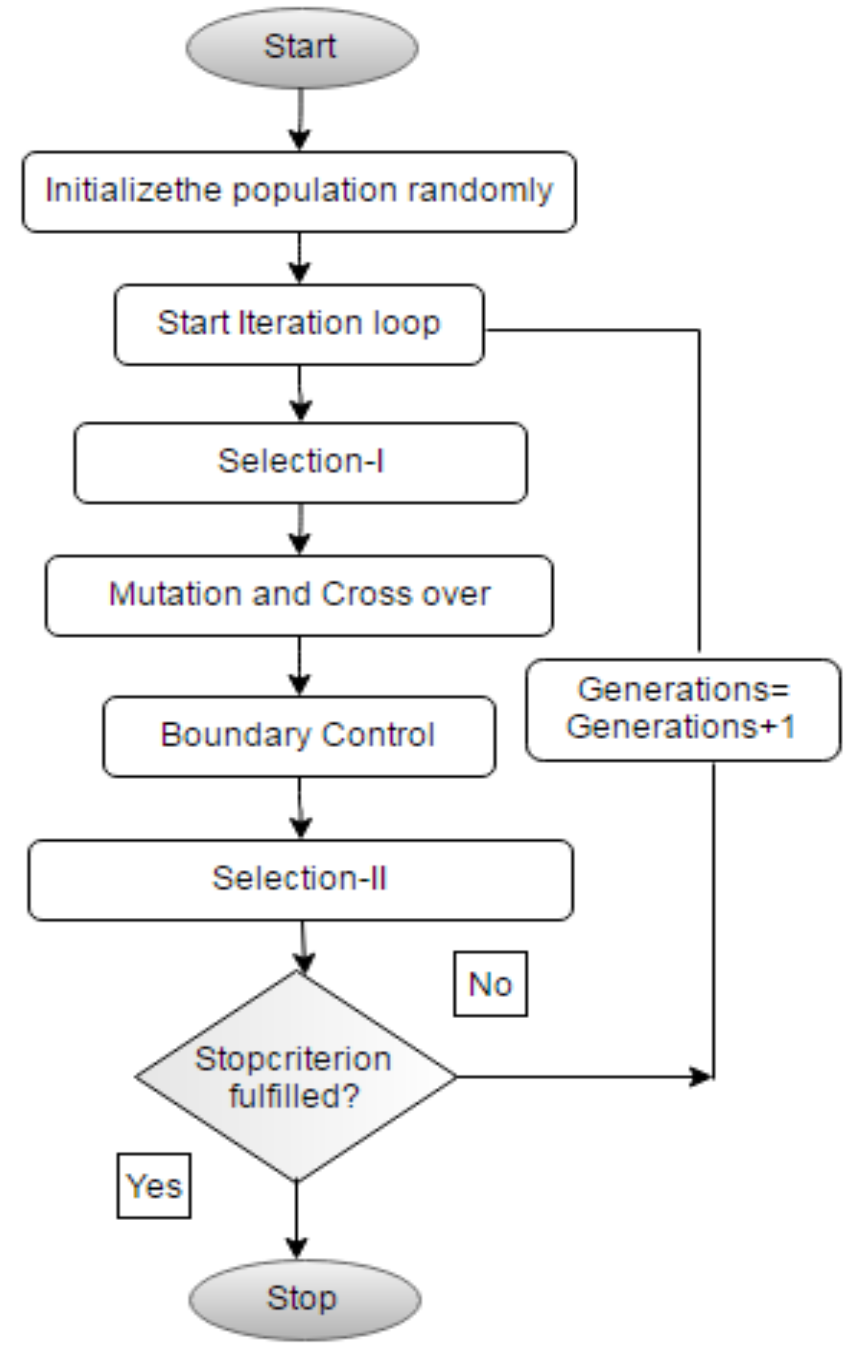

Fig. 2. Flow chart of BSA algorithm.

The CS algorithm, a new meta-heuristic algorithm imitating the animal behavior as described in [16-19] has two control parameters, one is population size $\mathrm{n}$ (or number of nests), and the other is $\mathrm{p}_{\mathrm{a}}$ (discovery rate). ABC algorithm, based on the honey bee foraging behavior as proposed in [20-22] has parameters, namely, colony size NP (employed bees+ onlooker bees) and the number of food source (FS). In our case, FS is set as half of the colony size (NP/2). A food source is considered as a potential solution for the problem case that needs to be optimized. Another control parameter is "limit". A food source is deemed to be discarded by the employed bee if its solution for the problem does not find any improvement within the set limits and this ends up in the employed bee allied with that corresponding food source becoming a scout. Flow charts of all the evolutionary algorithms are shown in Fig.2 to Fig.4. Details of the above three algorithms are available in the articles [14-22]. 


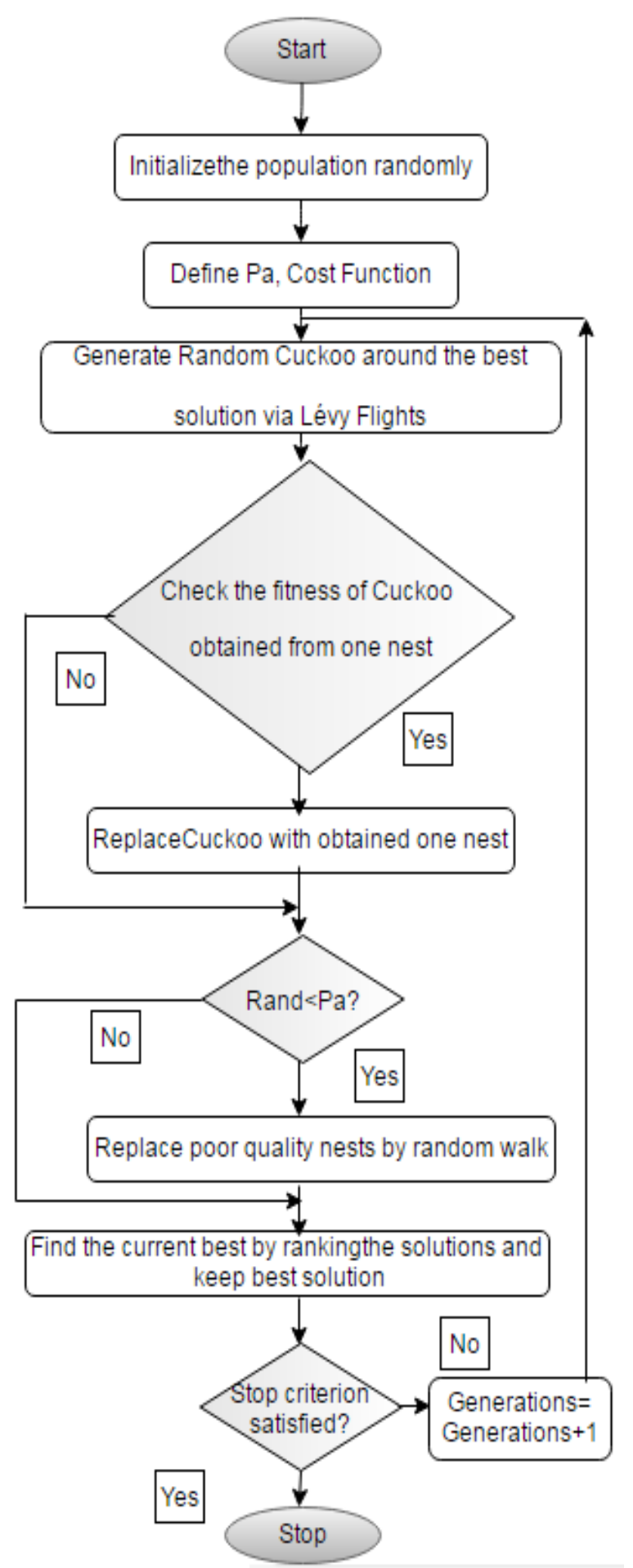

Fig. 3. Flow chart of CS algorithm.

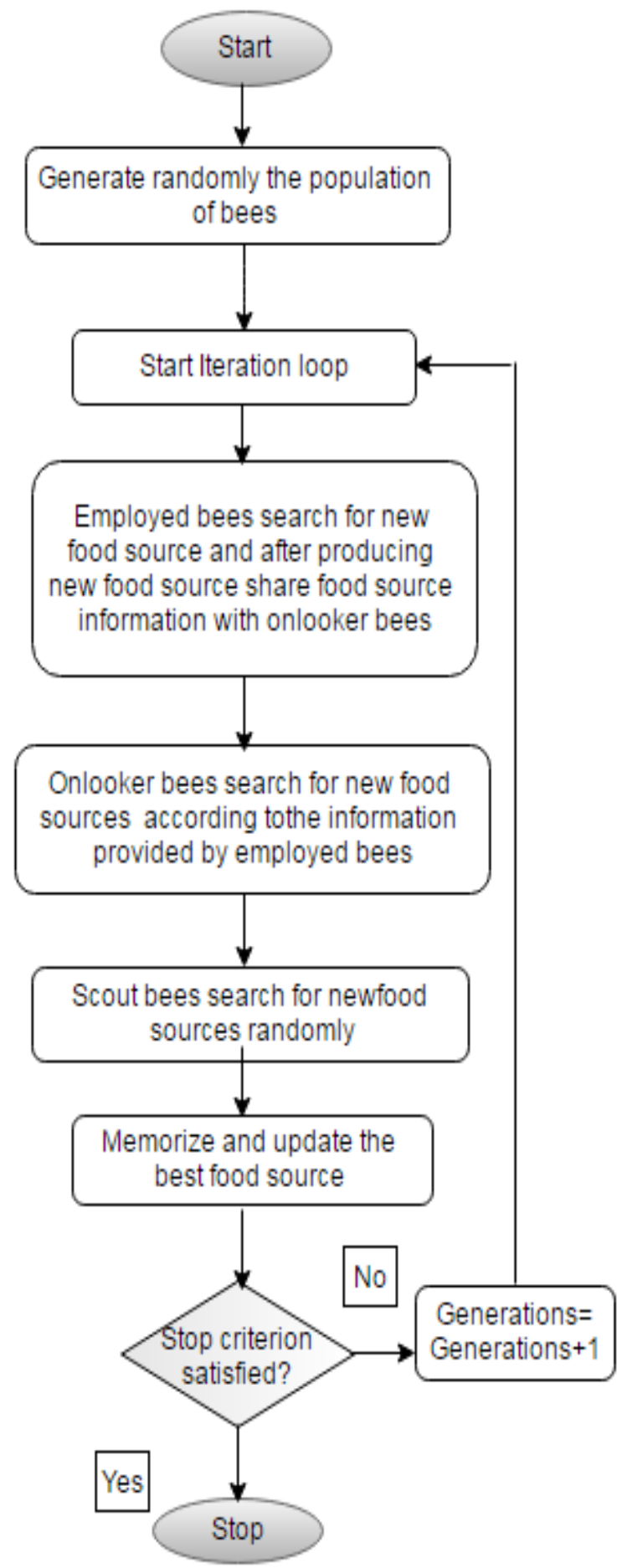

Fig.4. Flow Chart of ABC algorithm. 


\section{Simulated RESULTS}

Linear array of 26 and 100 parallel half-wave length antennas which are center-fed and uniformly spaced with an interval of $0.5 \lambda$ are considered along the $y$-axis in order to generate scanned far-field patterns in vertical plane ( $y-z$ plane). The excitation current amplitude distributions that are obtained using the algorithms is asymmetric. Each dipole's diameter is set to be $0.006 \lambda$ and the current distribution on each of the antenna array elements is assumed to be a sinusoidal one. The length-to-width ratio is 83.33 , which likely resembles a very thin sized dipole. Across each dipole in an array, because of mutual coupling effects, the active impedance varies with the beam scan angle. A 4096-point IFFT is used to speed up the process in these simulations.

The details of tuning parameters of all the evolutionary algorithms used in the problem are shown in the Table I For the purpose of comparison, the value of $k_{1}, k_{2}$ and $k_{3}$ or each of the optimization algorithms are chosen to get the best possible desired results from same fitness function. All the internal parameters are tuned by linear variation in between their minimum and maximum values or by trial and error method and then assigned a suitable value to the parameters for obtaining the scanned far-field pattern including the effects of mutual coupling with desired values.

For example 1: For the comparison performance of the used algorithms in terms of different antenna parameters as well as statistical parameters and for generation of power pattern, all the algorithms are run 20 times with 2000 iterations each and with a population size of 60 . One best scoring individual is generated after every run and a global best individual is considered as the best one out of such twenty best scoring individuals. A set of 26 current amplitude values is obtained by all the optimization techniques. Mean as well as the standard deviation of twenty best fitness values are calculated.

Simulations are done using Matlab software. The processing time that is involved in these simulations is measured with a PC with Intel core2 duo processor of clock frequency 2.93 $\mathrm{GHz}$ and $4 \mathrm{~GB}$ of RAM. Table II shows the obtained results using BSA, CS and ABC algorithms. Fig.5 shows the normalized current amplitude distribution. Fig.6 shows the normalized power pattern in $\mathrm{dB}$ scanned at $u=0.3$ using BSA, $\mathrm{CS}$ and ABC. Fig.7 shows global best fitness value versus iterations.

For example 2: the procedure that is followed in the above example 1 is utilized here except that the algorithms are run 100 times each with 1500 iterations and with a population size of 30. A set of 100 excitation current amplitude values is obtained by all the optimization techniques. Mean as well as the standard deviation of hundred best fitness values are then calculated.

Simulations are done using Matlab software. The computational time is measured with a PC with $\operatorname{Intel}(\mathrm{R})$ Core(TM) i5-4690 processor of clock frequency $3.50 \mathrm{GHz}$ and 4 GB of RAM. Table III shows the obtained results using BSA, CS and ABC algorithms. Fig. 8 shows the normalized current amplitude distribution. Fig. 9 shows the normalized power pattern in $\mathrm{dB}$ scanned at $u=0.45$. Fig. 10 shows global best fitness value versus iterations.
TABLE I

TUNING PARAMETERS OF ALL THE EVOLUTIONARY ALGORITHMS

\begin{tabular}{|c|c|c|c|c|c|c|}
\hline Example & \multicolumn{2}{|c|}{ Example1 (26 element) } & \multicolumn{3}{|c|}{ Example2 (100 element) } \\
\hline Parameters & BSA & CS & ABC & BSA & CS & ABC \\
\hline $\begin{array}{c}\text { Population } \\
\text { size }\end{array}$ & 60 & 60 & 60 & 30 & 30 & 30 \\
\hline Iteration & 2000 & 2000 & 2000 & 1500 & 1500 & 1500 \\
\hline Run number & 20 & 20 & 20 & 100 & 100 & 100 \\
\hline $\mathrm{K}_{1}, \mathrm{~K}_{2}, \mathrm{~K}_{3}$ & $22,1,8$ & $22,1,8$ & $22,1,8$ & $25,1,10$ & $25,1,10$ & $25,1,10$ \\
\hline Dim_Rate & 1 & - & - & 1 & - & - \\
\hline Scale factor & $3 *$ randn & - & - & $3 *$ randn & - & - \\
\hline $\mathrm{P}_{\mathrm{a}}$ & - & 0.25 & - & - & 0.25 & - \\
\hline Food number & - & - & 30 & - & - & 15 \\
\hline
\end{tabular}

TABLE II

COMPARATIVE OBTAINED RESULTS BY BSA, CS AND ABC FOR EXAMPLE 1

\begin{tabular}{|c|c|c|c|c|c|}
\hline $\begin{array}{c}\text { Design } \\
\text { Parameters }\end{array}$ & $\begin{array}{c}\text { Desired } \\
\text { Value }\end{array}$ & BSA & CS & ABC & Winner \\
\hline $\begin{array}{c}\text { Global Best } \\
\text { Fitness Value }\end{array}$ & - & 0 & 0 & 5.2656 & $\begin{array}{c}\text { BSA \& } \\
\text { CS }\end{array}$ \\
\hline $\begin{array}{c}\text { Standard } \\
\text { Deviation }\end{array}$ & - & 6.5037 & 3.9868 & 34.2951 & CS \\
\hline Mean & - & 5.2497 & 5.5859 & 77.3225 & BSA \\
\hline $\begin{array}{c}\text { Maximum } \\
\text { Active Scan } \\
\text { Impedance } \\
\text { (Ohm) }\end{array}$ & - & $78.29+$ & $90.02+$ & $85.92+$ & BSA \\
\hline $\begin{array}{c}\text { Side Lobe } \\
\text { Level (dB) }\end{array}$ & -25 & -25.09 & -25.01 & -24.53 & BSA \\
\hline $\begin{array}{c}\text { Maximum } \\
\text { Reflection } \\
\text { Coefficient }\end{array}$ & 0.33 & 0.2457 & 0.3011 & 0.2678 & BSA \\
\hline $\begin{array}{c}\text { Maximum } \\
\text { Wide Null } \\
\text { Depth in dB } \\
(u=0.6606 \text { to } \\
0.8071)\end{array}$ & -55 & -55.07 & -55.28 & -54.26 & CS \\
\hline $\begin{array}{c}\text { Computation } \\
\text { Time } \\
\text { (Seconds) }\end{array}$ & - & 8122.55 & 18973. & 8447.01 & BSA \\
\hline
\end{tabular}

TABLE III

COMPARATIVE OBTAINED RESULTS BY BSA, CS AND ABC FOR EXAMPLE 2

\begin{tabular}{|c|c|c|c|c|c|}
\hline $\begin{array}{c}\text { Design } \\
\text { Parameters }\end{array}$ & $\begin{array}{c}\text { Desired } \\
\text { Value }\end{array}$ & BSA & CS & ABC & Winner \\
\hline $\begin{array}{c}\text { Global Best } \\
\text { Fitness Value }\end{array}$ & - & 0 & 2.6546 & 355.5581 & BSA \\
\hline $\begin{array}{c}\text { Standard } \\
\text { Deviation }\end{array}$ & - & 11.0175 & 13.3128 & 148.4959 & BSA \\
\hline Mean & - & 14.0081 & 23.8119 & 773.98 & BSA \\
\hline $\begin{array}{c}\text { Maximum } \\
\text { Active Scan } \\
\text { Impedance } \\
\text { (Ohm) }\end{array}$ & - & $89.78-$ & $96.22-$ & $683.77-$ & BSA \\
\hline $\begin{array}{c}\text { Side Lobe } \\
\text { Level (dB) }\end{array}$ & -25 & -25.14 & -24.88 & -23.07 & BSA \\
\hline $\begin{array}{c}\text { Maximum } \\
\text { Reflection } \\
\text { Coefficient }\end{array}$ & 0.33 & 0.2873 & 0.3167 & 0.9476 & BSA \\
\hline $\begin{array}{c}\text { Maximum } \\
\text { Wide Null } \\
\text { Depth in dB } \\
(u=0.7582 \text { to } \\
0.9048)\end{array}$ & -50 & -50.12 & -48.46 & -33.96 & BSA \\
\hline $\begin{array}{c}\text { Computation } \\
\text { Time } \\
\text { (Seconds) }\end{array}$ & - & 6129.82 & 10457.10 & 6157.36 & BSA \\
\hline
\end{tabular}




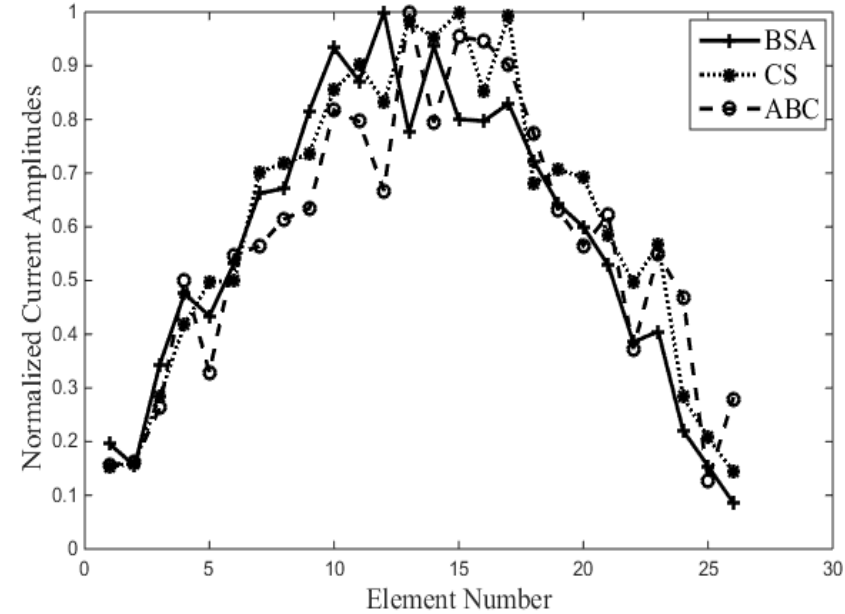

Fig. 5. Normalized current amplitude distribution versus element number for example 1.

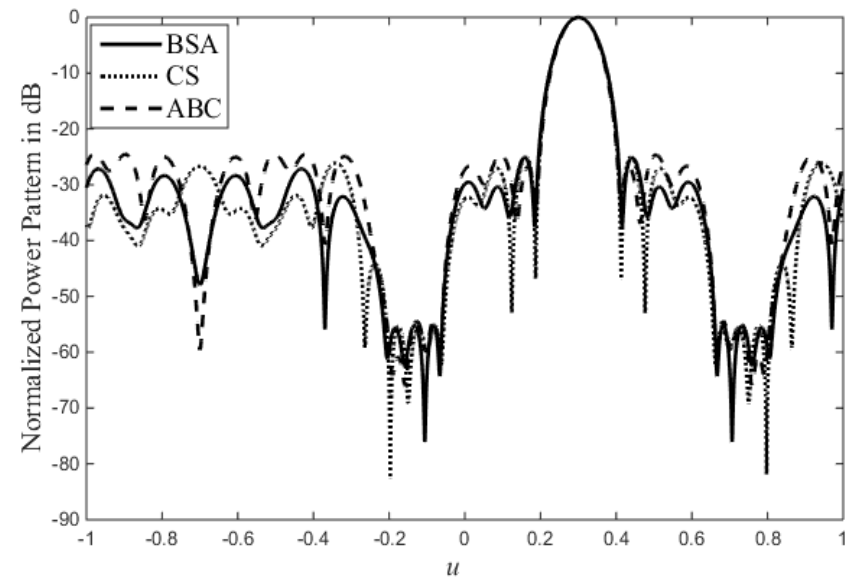

Fig. 6. Normalized power pattern in $\mathrm{dB}$ for a linear array of parallel halfwave length dipole antennas scanned at $u=0.3$ using BSA, CS and ABC with maximum wide null depth of $-55 \mathrm{~dB}$ from $u=0.6606$ to 0.8071 .

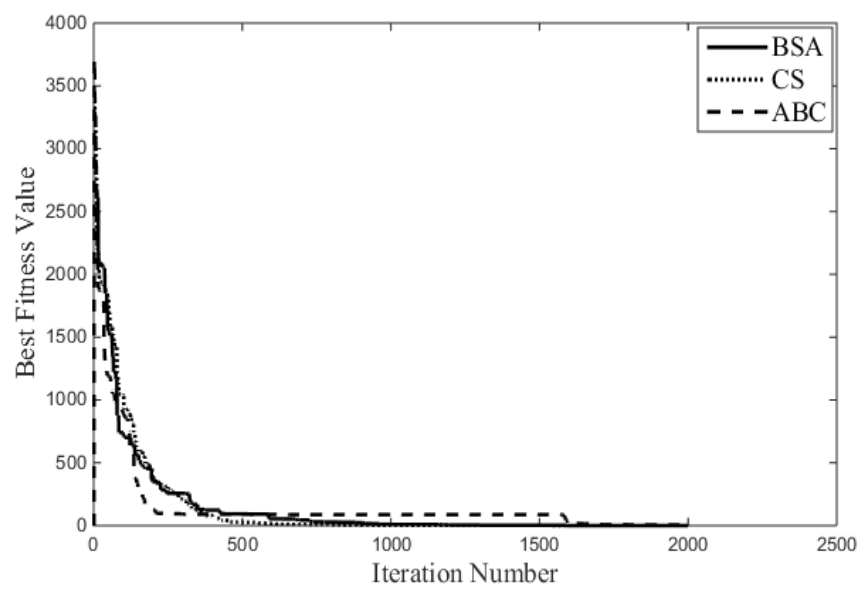

Fig. 7. Global best fitness value versus iteration number obtained by BSA, $\mathrm{CS}$ and $\mathrm{ABC}$ for generation of power pattern for example 1.

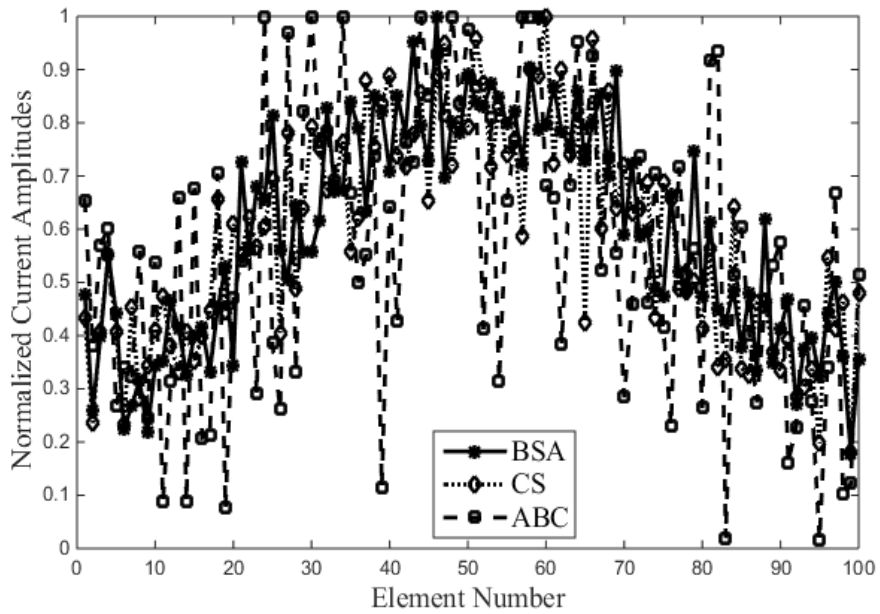

Fig. 8. Normalized current amplitude distribution versus element number for example 2.

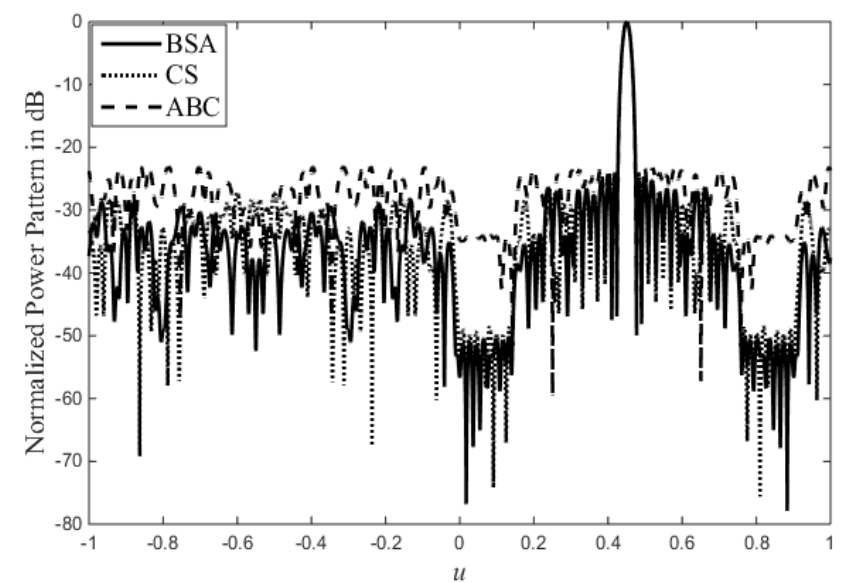

Fig. 9. Normalized power pattern in $\mathrm{dB}$ for a linear array of parallel halfwave length dipole antennas scanned at $u=0.45$ using BSA, CS and ABC with maximum wide null depth of $-50 \mathrm{~dB}$ from $u=0.7582$ to 0.9048 .

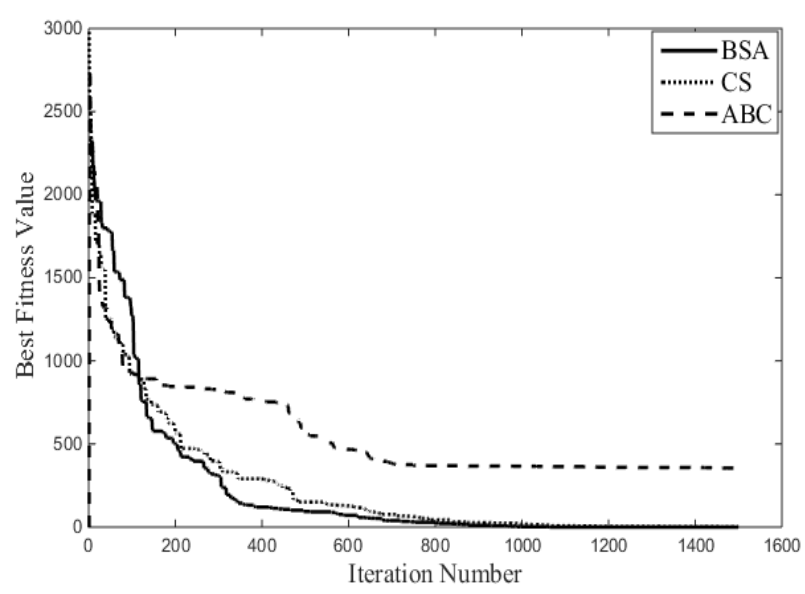

Fig. 10. Global best fitness value versus iteration number obtained by BSA, $\mathrm{CS}$ and $\mathrm{ABC}$ for generation of power pattern for example 2. 


\section{CONCLUSIONS}

This paper presented a comparative analysis of various evolutionary algorithms in terms on the antenna radiation pattern parameters as well as statistical parameters. The algorithms were tested in synthesis of the radiation pattern of a scanned linear array of parallel half-wavelength dipole antennas with fixed SLL and maximum reflection coefficient along with a wide null depth in the prescribed direction. From the obtained results of example1 (lower element), it was shown that all the algorithms are well suited for synthesis of power pattern in presence of mutual coupling. The desired and obtained values of antenna parameters such as SLL, maximum wide null depth and maximum reflection coefficient matched very closely with each other. BSA and CS were slightly better than $\mathrm{ABC}$ in reducing the peak SLL. CS gave more reflection coefficient than others. BSA and CS placed maximum wide null depth slightly better than $\mathrm{ABC}$. In the case of statistical parameters, the performance of all the algorithms matched closely with each other. BSA and CS showed better results than $\mathrm{ABC}$ in terms of global best fitness value, mean and standard deviation. Now from example2 (higher element), obtained results shown that BSA and CS are well suited for synthesis of power pattern in presence of mutual coupling than ABC. BSA provides good results for all antenna parameters as well as statistical parameters than $\mathrm{CS}$ and $\mathrm{ABC}$. The processing time of $\mathrm{CS}$ was more than $\mathrm{BSA}$ and $\mathrm{ABC}$, whereas BSA is slightly faster than ABC. A low value of maximum reflection coefficient ensured that impedance matching condition was greatly satisfied for all the array elements. Overall from the obtained results of both examples, it was found that BSA was more suitable for generation of scanned far-field pattern with the inclusion of mutual coupling than others.

\section{REFERENCES}

[1] C. A. Balanis, Antenna Theory: Analysis and Design, 2nd ed Singapore: John Wiley and Sons (Asia), 2003.

[2] R.L.Haupt, "Phase-only adaptive nulling with a genetic algorithm", IEEE Trans. on Antennas and Propagation, vol.45, no.6, pp.1009-1015, 1997.

[3] H. Steyskal, R.A. Shore, R.L. Haupt, "Methods for null control and their effects on the radiation pattern," IEEE Transactions on Antennas and Propagation, vol. 34, no. 3, pp.404-409, 1986.

[4] R.Vescovo, "Null synthesis by phase control for antenna arrays", Electronics Letter, vol.36, no.3, pp.198-199, 2000.

[5] H. Steyskal, "Simple method for pattern nulling by phase perturbation," IEEE Transactions on Antennas and Propagation, vol. 31, no. 1, pp $163-166,1983$.

[6] G.K. Mahanti, A. Chakraborty and S. Das, "Design of PhaseDifferentiated Reconfigurable Array Antennas with Minimum Dynamic Range Ratio," IEEE Antennas and Wireless Propagation Letters, vol.5, no.1, pp. 262-264, 2006.

[7] W. Boeringer Daniel and H. Werner Douglas, "Particle Swarm Optimization versus Genetic Algorithms for Phased Array Synthesis,' IEEE Transactions on Antennas and Propagation, vol. 52, no. 3, pp 771-779, 2004
[8] H.singh, H.L. Sneha, and R. M. Jha, "Mutual Coupling in Phased Arrays," International Journal of Antennas and Propagation, volume 2013, Article ID 348123, pp.1-23, 2013.

[9] M. Thevenot, C. Menudier, A. El Sayed Ahmad, G. Z. El Nashef, F. Fezai, Y. Abdallah, E. Arnaud, F. Torres, and T. Monediere, "Synthesis of Antenna Arrays and Parasitic Antenna Arrays with Mutual Couplings," International Journal of Antennas and Propagation, vol. 2012, pp.1-22, 2012.

[10] K. M. Lee and R. S. Chu, "Analysis of Mutual Coupling between a Finite Phased Array of Dipoles and Its Feed Network," IEEE Transactions on Antennas and Propagation, vol. 36, no. 12, 1681-1699, December 1988.

[11] B. K. J. C. Nauwelaers and A. R. Van de Capelle. "Integrals for the Mutual Coupling between Dipoles or Between Slots: With Without Complex Conjugate," IEEE Trans. on Antennas and Propagation, vol.36, no. 10, pp. 1375-1381, October 1988.

[12] F. Najib, R. Mohamad, and V. Patrick, "Effects of Mutual coupling in Phased Arrays with Butler Network Feed," in Proceedings of the 17th International Conference on Applied Electromagnetics and Communications, pp. 374-379, Dubrovnik, Croatia, October 2003.

[13] G.K.Mahanti, S. Das, A.Chakrabarty, J.C. Brégains, and F.Ares, "Design of Reconfigurable Array Antennas with Minimum Variation of Active Impedances," IEEE Antennas and Wireless Propagation Letters, vol. 5, no.1, pp. 541-544, 2006.

[14] P. Civicioglu, "Backtracking Search Optimization Algorithm for numerical optimization problems," Applied Mathematics and Computation, vol. 219, no. 15, pp. 8121-8144, 2013.

[15] R. Muralidaran, Vallavaraj.A, G.K. Mahanti and Ananya mahanti, "QPSO versus BSA for failure correction of linear array of mutually coupled parallel dipole antennas with fixed side lobe level and VSWR," Advances in Electrical Engineering, vol. 2014, Article ID 858290, pp. 1-7, September 2014.

[16] M.K. Ahirwal, Anil Kumar, and G.K. Singh, "EEG/ERP Adaptive Noise Canceller Design with Controlled Search Space (CSS) Approach in Cuckoo and Other Optimization Algorithms," IEEE/ACM Transactions on Computational Biology and Bioinformatics, vol. 10, no. 6, November/December 2013.

[17] Xin-She Yang and Suash Deb, "Cuckoo Search via Le'vy Flights," World Congress on Nature \& Biologically Inspired Computing (NaBIC), pp. 210-214, 2009.

[18] Xin-She Yang and Suash Deb, "Engineering Optimisation by Cuckoo Search," International journal on Mathematical Modelling and Numerical Optimisation, vol. 1, no. 4, pp. 330-343, 2010.

[19] R. Muralidaran, A.Vallavaraj, Hemant Patidar and G.K. Mahanti, "Failure correction of linear array of antenna with multiple null placement using cuckoo search algorithm," ICTACT Journal on Communication Technology, vol. 5, no.1, pp. 877-881, March 2014.

[20] D. Karaboga and B. Basturk, "A powerful and efficient algorithm for numerical function optimization: Artificial bee colony (ABC) algorithm," J. Global Optim., vol. 39, pp. 459-471, 2007.

[21] D. Karaboga and B. Akay, "A comparative study of artificial bee colony algorithm," Appl. Math. Compu. vol. 214, pp. 108-132, August 2009.

[22] A.Banharnsakun, T. Achalakul, and B. Sirinaovakun, "The best-so-far selection in artificial bee colony algorithm," Appl. Soft Comput., vol. 11, no. 2, pp. 2888-2901, Mar. 2011.

[23] L.L. Wang and D.G. Fang, and W.X. Sheng, "Combination of Genetic algorithm (GA) and Fast fourier transform (FFT) for Synthesis of Arrays, "Microwave and Optical Technology Letters, vol. 37, no. 1, pp. 56-59, April 2003.

[24] W. P. M. N. Keizer, "Fast Low Side lobe Synthesis for Large Planar Array Antennas Utilizing successive Fast Fourier Transforms of the Array Factor," IEEE Transactions on Antennas and Propagation, vol. 55, no.3, pp. 715-722, 2007.

[25] Will P. M. N. Keizer, "Low-Sidelobe Pattern Synthesis Using Iterative Fourier Techniques Coded in MATLAB," IEEE Antennas and Propagation Magazine, vol. 51, no.2, pp.137-150, April 2009. 